\title{
Energy-Efficient Routing Based on Dynamic Programming for Wireless Multimedia Sensor Networks (WMSNs)
}

\author{
Emansa Hasri Putra, Risanuri Hidayat, Widyawan, and I Wayan Mustika
}

\begin{abstract}
Wireless Multimedia Sensor Networks (WMSNs) advances can route multimedia applications from source nodes to a sink. However, they require energy efficiency and network lifetime due to limited power resources in the sensor nodes. This paper proposes an energy-efficient routing optimization for multimedia transmission in WMSNs. The optimization utilizes a routing algorithm based on the dynamic programming. The routing optimization algorithm selects intermediary nodes which have minimum energy above $60 \%$. Then, the priority selection of paths immediately finds neighboring nodes which have the greatest energy minimum. If there is the same minimum energy between the neighboring nodes, then the second priority selection is based on smaller link cost.
\end{abstract}

Keywords-wireless multimedia sensor networks (WMSNs), energy-efficient routing, optimization, dynamic programming.

\section{INTRODUCTION}

W IRELESS Multimedia Sensor Networks (WMSNs) is a network of wireless sensors for applications of audio, image, and video. The applications are transmitted by means of a multi-hop from the source node to the destination node (sink). Nodes of the WMSNs should be able to organize themselves dynamically and continuously maintain a connection between them. Therefore, the WMSNs has many advantages, namely cheap and easy implementation, a wide coverage area, easy to maintain, and a strong network connection.

The devices used in the WMSNs have limited resources, low processing speed, low storage capacity and limited bandwidth. Additionally, the WMSNs must operate for a long period. However, they only have battery-powered nodes. Therefore, the available energy resources of the nodes limit the operation of the overall network. To minimize energy consumption, most of the components of the devices including radios should be switched off most of the time. Another important characteristic of the WMSNs is that the nodes must have significant processing power as a group. The nodes should be able to organize and manage the network together. This is much more difficult than controlling the device individually. Furthermore, due to changes in the physical environment, the nodes also experience variations in the network connectivity and affect network protocols.

R. Hidayat, Widyawan, and I. W. Mustika are with the Department of Electrical Engineering and Information Technology, Universitas Gadjah Mada Indonesia (e-mail: risanuri@jteti.gadjahmada.edu, widyawan@ugm.ac.id, wmustika@ugm.ac.id).

E. H. Putra is with Department of Electrical Engineering, Politeknik Caltex Riau, Indonesia (email: emansa@pcr.ac.id)
The main design goal of the WMSNs is not only to transmit data from sources to a sink, but it also increases the network lifetime. The energy-efficient routing protocol can achieve this goal. Depending on the used applications, a wide variety of design and architecture has been applied in the Wireless Sensor Networks (WSNs) and the WMSNs. The performance depends on the routing protocol architecture and network design. The routing protocol operation can affect the energy used for data transmission.

Most of the energy consumption in the WSNs and WMSNs is spent on three main activities: sensing, data processing, and communication. All these factors are important when developing a protocol to the WMSNs. Communication between the nodes is a major component of energy consumption. Thus, the ongoing research in the WSNs and WMSNs is mostly concentrated on designing the routing protocols that use a little energy as possible during the communication nodes.

The main task of the routing protocols does not only find the shortest energy path from sources to a sink, but it also finds the most efficient way to extend the network lifetime. Continued use of the paths that has low energy often causes energy depletion of the nodes along this path. This can lead to partition or a broken network. Additionally, the routing protocols must consider the residual energy of the nodes as multimedia applications can drain the energy of the nodes quickly. Therefore, the routing protocols should choose the highest residual energy of neighboring nodes as the priority to route the multimedia applications [1-5].

In some literature, there are some studies related to multimedia transmission via the wireless multi-hop manner in unpredictable wireless networks. Multi-path and Multi-SPEED (MMSPEED) protocol use the IEEE 802.11e standard in the MAC layer to give priority in term of packet delivery guarantee. The downside of this protocol is underperforming to handle dead nodes and the overloaded network [6]. EnergyAware QoS protocol (EAQoS) utilizes a developed variant of Dijkstra's algorithm for routing optimization related to energy consumption and error rate. However, the algorithm requires complete information on the overall condition of the network to optimize the line that will be used [7]. Real-time Power-Aware Routing (RPAR) protocol also considers energyefficient approach for real-time communication. The downside of this protocol is the weak performance to handle dead nodes and busy networks [8]. The routing process has been mapped into a multi-stage decision process (dynamic programming) in the Wireless Mesh Network (WMN). The simulation results 
show that the new approach has a better performance regarding throughput and end-to-end delay [9]. The dynamic programming approach has been used to solve the problem of the crosslayer optimization design of channel coding, power allocation, and routing in the WSN [10]. This approach has also been used to guarantee Quality of Service (QoS) of video transmission and energy efficiency in Wireless Video Sensor Networks (WVSNs) [11]. A cross-layer design method has been applied to the optimization process of H.264/SVC video delivery via WLANs [12], and the WMSNs [13]. AOMDV routing protocols have demonstrated better performance than AODV. Then, the AOMDV protocol is combined with H.264/AVC and the IEEE 802.15.4 to maximize the lifetime of the WMSN [14].

However, to achieve an efficient-routing for multimedia transmission is a common challenge in the WMSNs as they have particular characteristics. The routing algorithm on the WMSNs should be able to find the shortest route between the nodes while taking into account the residual energy of the nodes traversed. In this paper, the routing optimization in the WMSNs is investigated. The routing optimization considers the residual energy of the nodes traversed and employs the dynamic programming to model it.

The rest of this paper is organized as follows. Section II explains the WMSNs. Section III explains the Dynamic Programming. Section IV explains Proposed Routing Algorithm. Section V explains Results and Discussion. Finally, the Conclusions are presented in Section VI.

\section{Wireless Multimedia Sensor Network (WMSN)}

The Wireless Multimedia Sensor Network (WMSN) is a wireless-based system that will read data from multimedia sensors. Due to technological developments have made it possible for the camera sensor installed in sensor nodes. Therefore, the sensor nodes are able to transmit various multimedia applications such as audio and video. The WMSN can be divided into several clusters and each cluster consists of sensor nodes. The results of the reading of the sensors will be delivered in wireless multi-hop and displayed in a sink.

Fig. 1 consitutes a general models of the WMSN. The model consists of three different sensor networks, namely singlelayer network of different sensor nodes, a single-layer network of similar sensor nodes, and multi-layers of different sensor network nodes. In the single-layer network of different sensor nodes, applications of audio, videos, and scalar of sensor nodes are sent to the center cluster head. Then the center cluster head will act as a processing hub. The results of the processing in the cluster head will be sent to the wireless gateway over the multi-hop manner.

In the single-layer network of similar sensor nodes, each sensor node has the same capabilities and tasks. Multimedia applications of the sensor nodes will be sent through neighboring nodes to the gateway over the wireless multi-hop manner. In the multi-layer network of different sensor nodes, Each layer has a specific task. Sensors with low power and limited resources only work for simple tasks, whereas sensors with the high power devices and rich resources function to more

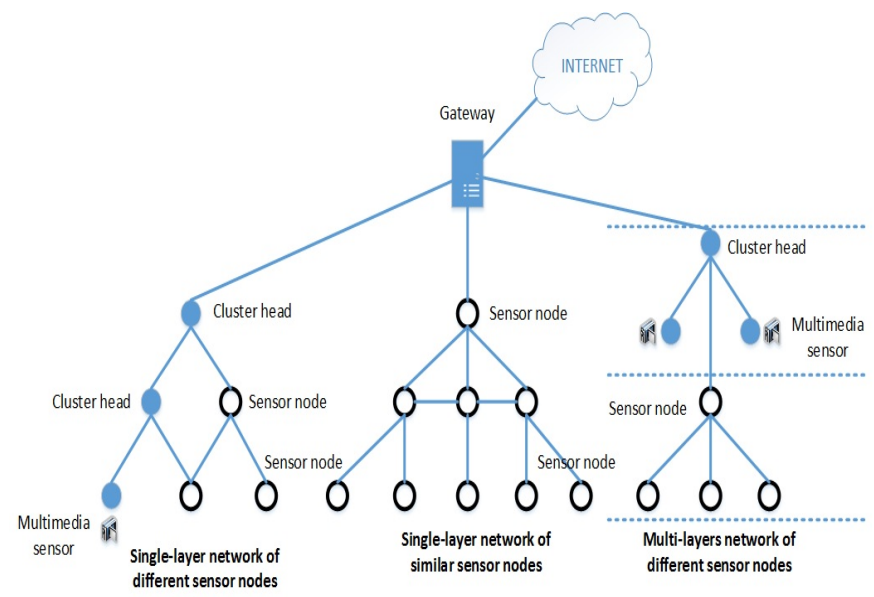

Fig. 1. A general model of Wireless Multimedia Sensor Networks (WMSNs)

complex tasks. Processing and storage of data is distributed through all different levels [1].

\section{DynAmiC PROgRAMming}

A dynamic programming optimization is an approach that transforms a complex problem in a specific sequence or parts of smaller problems. The dynamic programming as an optimization method has several advantages:

1) The process of solving complex problems into smaller problem parts so that the sources of these problems become easier to understand.

2) The method of dynamic programming can solve a wide range of optimization problems that tends to be more flexible than other optimization methods.

3) Procedures for the calculation of dynamic programming involve the analysis of each variable stated in each decision stage.

4) Dynamic programming can customize the calculations according to problems size and keep doing the calculation process step by step in a complete and comprehensive way.

For the optimization problem in general, the goal of this optimization approach is to maximize the functions of the decision or to minimize the functions of the cost of the decision stage, $f_{n}\left(d_{n}, s_{n}\right)$. Thus for state $\left(s_{n}\right)$ of stage $n$, the optimal-value function, $v_{n}\left(s_{n}\right)$, of the optimization problem is [15]:

$$
\begin{aligned}
v_{n}\left(s_{n}\right) & =\operatorname{Min}\left[f_{n}\left(d_{n}, s_{n}\right)+f_{n-1}\left(d_{n-1}, s_{n-1}\right)+\ldots\right. \\
& \left.+f_{0}\left(d_{0}, s_{0}\right)\right]
\end{aligned}
$$

Subject to:

$$
\begin{array}{ll}
s_{m-1}=t_{m}\left(d_{m}, s_{m}\right) & (m=1,2, \ldots, n), \\
d_{m} \in D_{m} & (m=1,2, \ldots, n) .
\end{array}
$$

The above formula can also be simplified into:

$$
v_{n}\left(s_{n}\right)=\operatorname{Min}\left\{f_{n}\left(d_{n}, s_{n}\right)+v_{n-1}\left[t_{n}\left(d_{n}, s_{n}\right)\right]\right\}
$$

Subject to:

$$
d_{n} \in D_{n}
$$




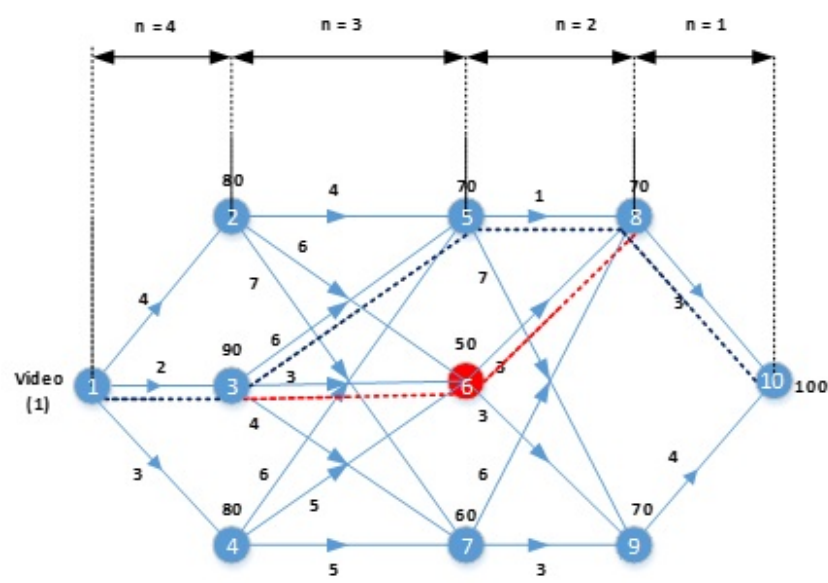

Fig. 2. A proposed energy-efficient routing based on dynamic programming

\section{Proposed Routing Algorithm}

A proposed energy-efficient routing based on the dynamic programming is shown in Fig. 2. There are sensor nodes 1, 2, $3,4,5,6,7,8,9$, and 10. Each node has a certain distance (link cost) to the neighboring nodes. The sensor node 1 will transmit video applications into the sensor node 10, through intermediary nodes by choosing the shortest distance or link cost. After the shortest route is selected, packets of videos will be passed to the nodes on the condition that the nodes must have energy left over $50 \%(\mathrm{E}>50)$.

By using a mathematical model of the dynamic programming, the network above is divided into four stages $(n)$, starting from the rear, $n=1$. Thus, the mathematical model of the dynamic programming for the network is:

$$
\begin{aligned}
v_{4}\left(s_{4}\right) & =\operatorname{Min}\left[f_{4}\left(d_{4}, s_{4}\right)+f_{3}\left(d_{3}, s_{3}\right)+f_{2}\left(d_{2}, s_{2}\right)\right. \\
& \left.+f_{1}\left(d_{1}, s_{1}\right)\right]
\end{aligned}
$$

To get to the sensor node 10 as illustrated in Fig. 2, the available sensor nodes are 8 , and 9 that each node has a link cost of 3 and 4 as shown in Table 1 . The result of the first stage is the sensor node 10 for the sensor nodes 8 and 9 .

To get to the sensor nodes 8 and 9 as illustrated in Fig. 2, the available sensor nodes are 5, 6, and 7. The sensor node 5 goes to the sensor node 10 through the sensor node 8 , the link cost is $1+3=4$. The sensor node 5 goes to the sensor node 10 through the sensor node 9 , the link cost is $7+4=$ 11. From the sensor node 5 goes to the sensor node 10 , a link cost selection is decided from the sensor node 8 because the link cost has the lowest value as shown in Table 2 . The results of other decisions from the sensor nodes 6 and 7 to the sensor node 10 are shown in Table 2 .

To get to the sensor nodes 5, 6, and 7 as illustrated in Fig. 2 , the available sensor nodes are 2,3 , and 4 . The sensor node 2 goes to the sensor node 10 through the sensor node 5 , the link cost is $4+4=8$, and the sensor node 2 goes to the sensor node 10 through the sensor node 6 , the link cost is $6+6=12$. Then, the sensor node 2 goes to the sensor node 10 through the sensor node 7 , the link cost is $7+7=14$. From the sensor node 3 goes to the sensor node 10, a link cost selection is decided from the sensor node 5 because the link cost has the

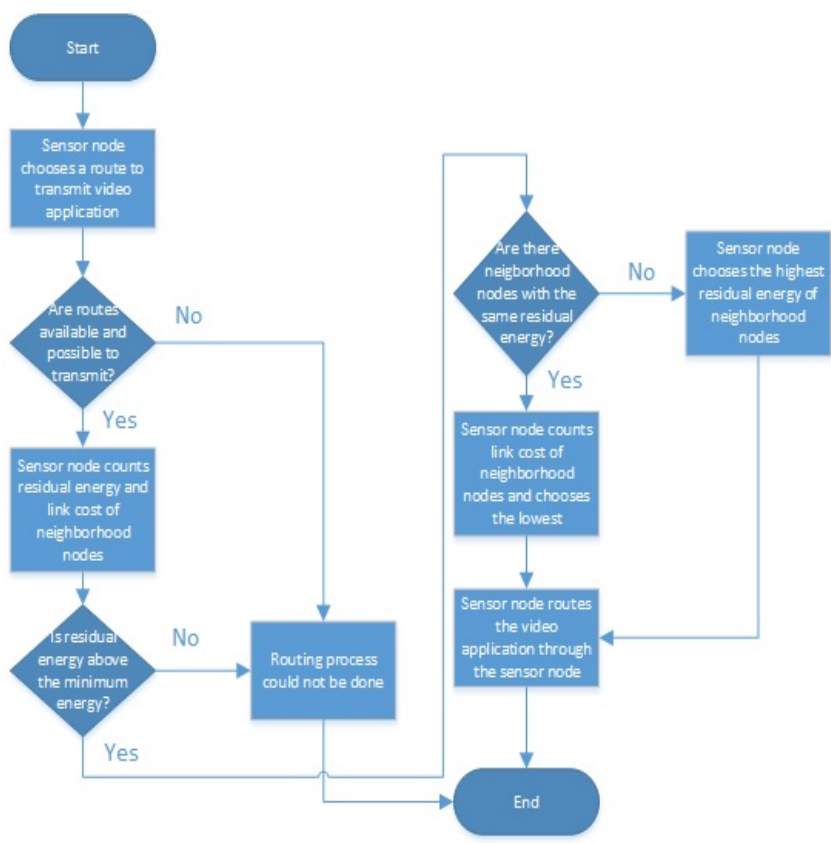

Fig. 3. The proposed energy-efficient routing algorithm

value above the minimum energy $(>50 \%)$ and the second lower link cost as shown in Table 3. Even the sensor node 6 has the lowest link cost, but it would not be selected as the minimum energy must be above $50 \%$. The results of other decisions are shown in Table 3 from the sensor nodes 2, 3, and 4 to the sensor node 10 .

To get to the sensor nodes 2, 3, and 4 as illustrated in Fig. 2 , the available sensor node is 1 which each sensor node has a link cost of 12, 10, and 13 as shown in Table 4 . The result of the first phase of the sensor nodes 8 and 9 is the sensor node 10.

Regardless of the minimum energy factor of the sensor node 6 , then the selection is the shortest route:

Sensor nodes $\mathbf{1}-\mathbf{3}-\mathbf{6}-\mathbf{8}-\mathbf{1 0}$

As the minimum energy must be above $50 \%$, the sensor node 6 does not have enough minimum energy to route the video packets. Therefore, the shortest route and the rest of the corresponding energy requirement are:

Sensor nodes 1 - 3-5 - 8 - 10

The process of the energy-efficient routing based on the dynamic programming is explained in Fig. 3. When a sensor node wants to send a video application, firstly the sensor node must determine whether the routes are available to pass the video packets or not. If the routes have been provided, the sensor node starts calculating the residual energy and link cost of the nodes that are needed to be passed. Then, the sensor node determines whether the residual energy of the nodes that will be passed over the minimum energy requirement or not. If they all are the above minimum energy requirement, then the sensor node will select the sensor node with the highest residual energy. If there is no sensor node with the highest residual energy, then the sensor node will calculate the link cost and then select the lowest link cost. 
TABLE 1

THE RESULTS OF THE FIRST STAGE

\begin{tabular}{|c|c|c|c|}
\hline $\boldsymbol{S}_{\mathbf{1}} \backslash \boldsymbol{d}_{\mathbf{1}}$ & $\boldsymbol{f}_{\boldsymbol{n}}$ & Min link cost & $\boldsymbol{v}_{\boldsymbol{1}}\left(\boldsymbol{s}_{\mathbf{1}}\right)$ \\
\hline $\mathbf{8}$ & 3 & 3 & $\mathbf{1 0}$ \\
\hline $\mathbf{9}$ & 4 & 4 & $\mathbf{1 0}$ \\
\hline
\end{tabular}

TABLE 2

THE RESULTS OF THE SECOND STAGE

\begin{tabular}{|c|c|c|c|c|}
\hline $\boldsymbol{S}_{\mathbf{2}} \backslash \boldsymbol{d}_{\mathbf{2}}$ & $\mathbf{8}$ & $\mathbf{9}$ & Min link cost & $\boldsymbol{v}_{\mathbf{2}}\left(\boldsymbol{s}_{\mathbf{2}}\right)$ \\
\hline $\mathbf{5}$ & $1+3=4$ & $7+4=11$ & 4 & $\mathbf{8}$ \\
\hline $\mathbf{6}$ & $3+3=6$ & $3+4=7$ & 6 & $\mathbf{8}$ \\
\hline $\mathbf{7}$ & $6+3=9$ & $3+4=7$ & 7 & $\mathbf{9}$ \\
\hline
\end{tabular}

TABLE 3

THE RESULTS OF THE THIRD STAGE

\begin{tabular}{|c|c|c|c|c|c|}
\hline $\boldsymbol{S}_{\mathbf{3}} \backslash \boldsymbol{d}_{\mathbf{3}}$ & $\mathbf{5}$ & $\mathbf{6}$ & $\mathbf{7}$ & Min link cost & $\boldsymbol{v}_{\mathbf{3}}\left(\boldsymbol{s}_{\mathbf{3}}\right)$ \\
\hline $\mathbf{2}$ & $4+4=8$ & $6+6=12$ & $7+7=14$ & 8 & $\mathbf{5}$ \\
\hline $\mathbf{3}$ & $6+4=10$ & $3+6=9$ & $4+7=11$ & $10(9)$ & $\mathbf{5}(\mathbf{6})$ \\
\hline $\mathbf{4}$ & $6+4=10$ & $5+6=11$ & $5+7=12$ & 10 & $\mathbf{5}$ \\
\hline
\end{tabular}

TABLE 4

THE RESULTS OF THE FOURTH STAGE

\begin{tabular}{|c|c|c|c|c|c|}
\hline $\boldsymbol{S}_{\mathbf{4}} \backslash \boldsymbol{d}_{\mathbf{4}}$ & $\mathbf{2}$ & $\mathbf{3}$ & $\mathbf{4}$ & Min link cost & $\boldsymbol{v}_{\mathbf{4}}\left(\boldsymbol{s}_{\mathbf{4}}\right)$ \\
\hline $\mathbf{1}$ & $4+8=12$ & $2+8=10$ & $3+10=13$ & 10 & $\mathbf{3}$ \\
\hline
\end{tabular}
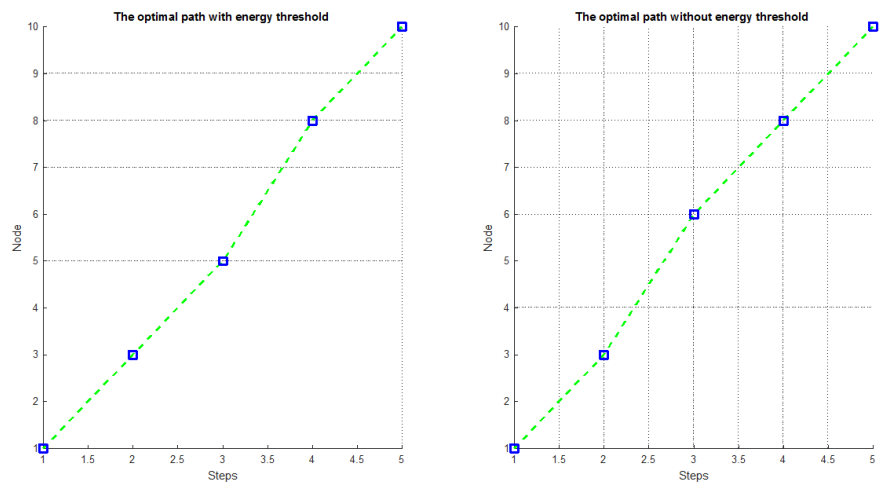

Fig. 4. The routing optimization result of the second scheme (left) and the first scheme (right) for 10 sensor nodes

\section{RESUlTS AND DiscusSiON}

The proposed energy-efficient routing based on the dynamic programming is simulated as shown in Fig. 4. The first simulation consists of 10 sensor nodes. Each has a different energy minimum (energy threshold). The dynamic programming algorithm is used to find the shortest route from the sensor node 1 to the sensor node 10 as described in section 4. In the first scheme, the routing optimization only focuses on the shortest distance regardless of the minimum energy of the nodes traversed. Then, in the second scheme, it should pay attention to the minimum energy of the nodes traversed. Then, it selects the node that has the largest minimum energy. If there are two or more nodes having the same minimum energy, the smallest link cost will be selected. The second simulation involves 45 sensor nodes that the schemes are the same as the first simulation.
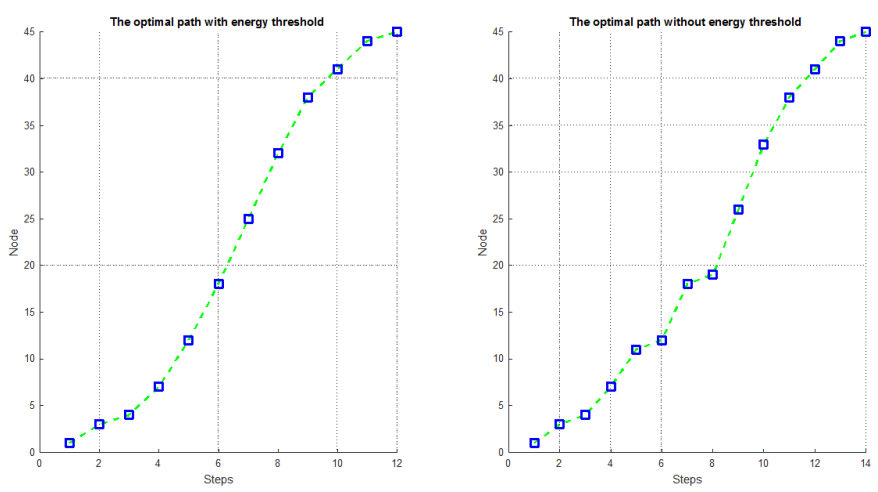

Fig. 5. The routing optimization result of the second scheme (left) and the first scheme (right) for 45 sensor nodes

The first simulation results are shown in Fig. 4. The graph to the left shows the results of the second scheme that focus on energy-efficiency and the shortest path. The nodes that are passed are 1-3-5-8-10. Then, the total link costs are 12. The graph to the right shows the routing optimization results of the first scheme that focus only on the shortest path. The nodes that are passed are 1-3-6-8-10. Then, the total link costs are 11 .

The second simulation results are shown in Fig. 5. The graph to the left shows the routing optimization results of the second scheme that focus on energy-efficiency and the shortest path. Nodes that are passed are 1-3-4-7-12-18-25-3238-41-44-45. Then, the total link costs are 27 . The graph to the right shows the routing optimization results of the first scheme that focus only on the shortest path. The nodes that are passed are 1-3-4-7-11-12-18-19-26-33-38-41-44-45. Then, the total link costs are 26. 


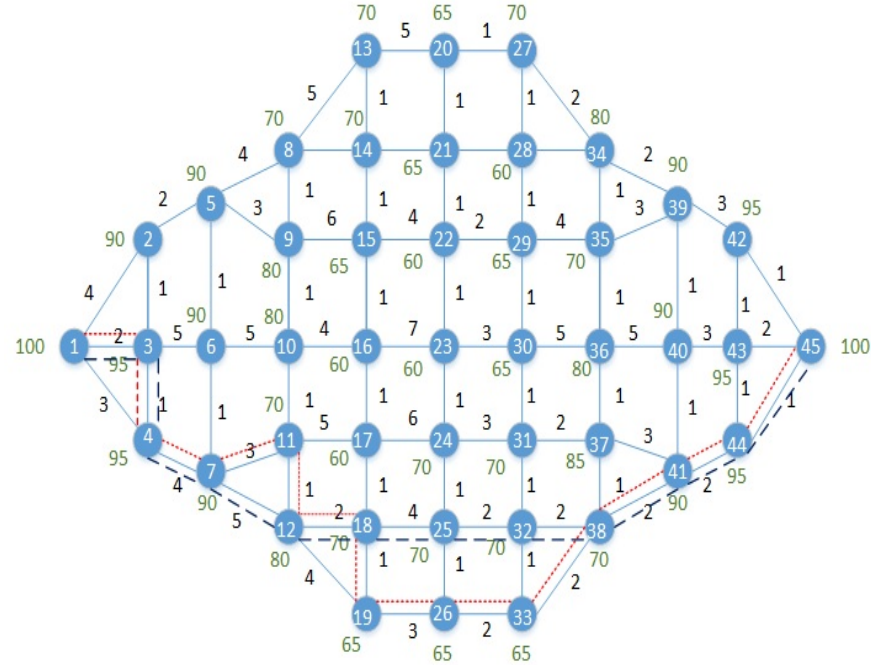

Fig. 6. The energy-efficient routing optimization results from sensor node 1 to sensor node 45

The routing optimization results from the sensor node 1 to the sensor node 45 are indicated in Fig. 6. The red lines show the routing optimization results of the first scheme that focus only on the shortest path. The blue line shows the routing optimization results of the second scheme that focus on energy-efficiency and the shortest path. The first routing optimization algorithm will select intermediary nodes which have the minimum energy above $60 \%$. Then, the selection of paths to be traversed. Then, the priority immediately finds neighboring nodes which have the greatest minimum energy. If there is the same minimum energy between the neighboring nodes, then the second priority is the smaller link cost.

\section{CONCLUSION}

1) The optimization process of the routing protocols in Wireless Multimedia Sensor Networks (WMSNs) that focuses on the energy efficiency is achieved by using the dynamic programming.

2) The simulation results show that the minimum energy (energy threshold) of the sensor node becomes the main requirement before selecting the shortest route (the shortest path) of the dynamic programming.

\section{REFERENCES}

[1] I. F. Akyildiz, T. Melodia, and K. R. Chowdhury, A survey on wireless multimedia sensor networks, Comput. Networks, vol. 51, no. 4, pp. 921960, 2007. http://dx.doi.org/ 10.1109/MWC.2007.4407225

[2] S. Misra, M. Reisslein, and G. Xue, A survey of multimedia streaming in wireless sensor networks, IEEE Commun. Surv. Tutorials, vol. 10, no. 4 , pp. 1839, 2008. http://dx.doi.org/10.1109/SURV.2008.080404

[3] S. Ehsan and B. Hamdaoui, A Survey on Energy-Efficient Routing Techniques with QoS Assurances for Wireless Multimedia Sensor Networks, IEEE Commun. Surv. Tutorials, vol. 14, no. 2, pp. 265278, Jan. 2012 http://dx.doi.org/10.1109/surv.2011.020211.00058

[4] N. A. Pantazis, S. A. Nikolidakis, and D. D. Vergados, EnergyEfficient Routing Protocols in Wireless Sensor Networks: A Survey, IEEE Commun. Surv. Tutorials, vol. 15, no. 2, pp. 551591, 2013. http://dx.doi.org/10.1109/surv.2012.062612.00084

[5] J. N. Al-Karaki and a E. Kamal, Wireless Sensor Networks Routing Techniques in Wireless Sensor Networks: A Survey, IEEE Wirel. Commun., vol. 11, no. December, pp. 628, 2004 http://dx.doi.org/10.1109/mwc.2004.1368893

[6] Felemban, Emad, Chang-Gun Lee, and Eylem Ekici. MMSPEED: Multipath multi-SPEED protocol for QoS guarantee of reliability and timeliness in wireless sensor networks. IEEE transactions on mobile computing 6 (2006): 738-754. http://dx.doi.org/10.1109/tmc.2006.79

[7] Akkaya, Kemal, and Mohamed Younis. An energy-aware QoS routing protocol for wireless sensor networks. Distributed Computing Systems Workshops, 2003. Proceedings. 23rd International Conference on. IEEE, 2003. http://dx.doi.org/10.1109/icdcsw.2003.1203636

[8] Chipara, Octav, et al. Real-time power-aware routing in sensor networks Quality of Service, 2006. IWQoS 2006. 14th IEEE International Workshop on. IEEE, 2006. http://dx.doi.org/10.1109/iwqos.2006.250454

[9] Y. Qin and R. Zhu, Efficient Routing Algorithm Based on Decision-making Sequence in Wireless Mesh Networks, J. Networks, vol. 7, no. 3, pp. 502509, 2012. http://dx.doi.org/10.4304/jnw.7.3.502-509

[10] L. Song, Y. Zhang, R. Yu, W. Yao, and Z. Wu, Cross-Layer Optimized Routing for Wireless Sensor Networks Using Dynamic Programming, no. X, 2009. http://dx.doi.org/10.1109/icc.2009.5198875

[11] Fallahi, Afshin, and Ekram Hossain. A dynamic programming approach for OoS-aware power management in wireless video sensor networks. Vehicular Technology, IEEE Transactions on 58.2 (2009): 843854. http://dx.doi.org/10.1109/tvt.2008.927714

[12] M. Li, Z. Chen, and Y. P. Tan, Cross-layer optimization for SVC video delivery over the IEEE 802.11e wireless networks, J. Vis Commun. Image Represent., vol. 22, no. 3, pp. 284296, 2011. http://dx.doi.org/10.1016/j.jvcir.2011.01.002

[13] E. H. Putra, R. Hidayat, Widyawan, and I. W. Mustika, CrossLayer Design of Wireless Multimedia Sensor Network Based on IEEE 802.11e EDCA and H.264/SVC, in 2015 International Conference on Science in Information Technology (ICSITech), 2015 http://dx.doi.org/10.1109/icsitech.2015.7407779

[14] A. Ben Ammar, O. Bouattay, A. Dziri, M. Terre, and H. Youssef, Performance analysis of AODV and AOMDV over SMAC and IEEE 802.15.4 in Wireless Multimedia Sensor Network, in 2015 International Wireless Communications and Mobile Computing Conference (IWCMC), 2015 , no. 1, pp. 14641468. http://dx.doi.org/10.1109/iwcmc.2015.7289298

[15] H. Bradley, Chapter 11 - Dynamic Programming, Appl. Math. Program., vol. 26, no. 104 , pp. 320 362, 1977. 